\title{
ON SOME NONLINEAR PARTIAL DIFFERENTIAL EQUATIONS INVOLVING THE "1"-LAPLACIAN AND CRITICAL SOBOLEV EXPONENT
}

\author{
FranÇOISE DEMENGel ${ }^{1}$
}

\begin{abstract}
Let $\Omega$ be a smooth bounded domain in $\mathbf{R}^{n}, n>1$, let $a$ and $f$ be continuous functions on $\bar{\Omega}, 1^{\star}=\frac{n}{n-1}$. We are concerned here with the existence of solution in $B V(\Omega)$, positive or not, to the
\end{abstract} problem:

$$
\left\{\begin{aligned}
-\operatorname{div} \sigma+a(x) \operatorname{sign} u & =f|u|^{1^{\star}-2} u \\
\sigma \cdot \nabla u & =|\nabla u| \text { in } \Omega \\
u \text { is not identically zero, } & -\sigma \cdot n(u)=|u| \text { on } \partial \Omega .
\end{aligned}\right.
$$

This problem is closely related to the extremal functions for the problem of the best constant of $W^{1,1}(\Omega)$ into $L^{\frac{N}{N-1}}(\Omega)$.

Résumé. On s'intéresse aux solutions dans $B V(\Omega)$, non identiquement nulles au problème

$$
\begin{gathered}
-\operatorname{div}\left(\sigma+a(x) \operatorname{sign} u=f|u|^{1^{\star}-2} u\right. \\
\sigma \cdot \nabla u=|\nabla u| \\
-\sigma \cdot \vec{n} u=|u| \operatorname{sur} \partial \Omega
\end{gathered}
$$

(où $\Omega$ est un ouvert borné régulier de $\mathbf{R}^{N}$.)

AMS Subject Classification. 35J70.

Received July 8, 1998. Revised September 7, 1999.

\section{INTRODUCTION}

Let $\Omega$ be a bounded open subset of $\mathbf{R}^{n}$, whose boundary is piecewise $\mathcal{C}^{1}$, let $a$ and $f$ be smooth (at least continuous on $\bar{\Omega}), f$ being positive somewhere, $a$ being such that there exists some constant $c>0$ such that for all $u \in W_{0}^{1,1}(\Omega)$,

$$
|\nabla u|_{L^{1}(\Omega)}+\int_{\Omega} a|u| \geq c|| u \|_{W^{1,1}(\Omega)} .
$$

We look for $u \in W_{0}^{1,1}(\Omega)$, which satisfies:

Keywords and phrases: BV functions, best constant for Sobolev embeddings.

1 Université de Cergy-Pontoise, Département de Mathématiques, Site de Saint-Martin, 2 avenue Adolphe Chauvain, 95302 Cergy-Pontoise Cedex, France; e-mail: Francoise.DEMENGEL@math.u-cergy.fr 


$$
\left\{\begin{aligned}
-\operatorname{div} \sigma+a(x) \operatorname{sign} u & =f|u|^{1^{\star}-2} u \\
\sigma & \in L^{\infty}\left(\Omega, \mathbf{R}^{n}\right), \\
\sigma \cdot \nabla u & =|\nabla u| \\
u \text { is not identically zero, } u & =0 \text { on } \partial \Omega
\end{aligned}\right.
$$

where $1^{\star}=\frac{n}{n-1}$ is the critical Sobolev exponent for the embedding of $W^{1,1}(\Omega)$ into $L^{q}(\Omega)$, and sign $u$ denotes some $L^{1}$ function defined as:

$$
\operatorname{sign} u u=|u| \text {. }
$$

Later we shall impose to $u$ in (1.2) to be non negative, and we shall replace sign $u$ by $\operatorname{sign}^{+} u$ defined as

$$
\operatorname{sign}^{+} u \cdot u=u^{+}
$$

In order to find solutions to (1.2) one can consider the following minimisation problem

$$
\inf _{\left\{u \in W_{0}^{1,1}(\Omega), \int_{\Omega} f|u|^{1^{\star}}=1\right\}} \int_{\Omega}|\nabla u|+\int_{\Omega} a(x)|u| .
$$

We denote by $\lambda(\Omega, a, f)$ the value of this infimum. Indeed, if $v \in W_{0}^{1,1}(\Omega)$ realizes the minimum defined in (1.4), it is a non trivial solution of

$$
\left\{\begin{aligned}
-\operatorname{div} \sigma+a(x) \operatorname{sign} v & =\lambda(\Omega, a, f) f|v|^{1^{\star}-2} v \\
\sigma & \in L^{\infty}\left(\Omega, \mathbf{R}^{n}\right) \\
\sigma . \nabla v & =|\nabla v| \\
v & =0 \text { on } \partial \Omega
\end{aligned}\right.
$$

then $u=(\lambda(\Omega, a, f))^{\frac{1}{n-1}} v$ satisfies $(1.2)$.

In a previous paper [6] (see also [13]), we were concerned with the existence of solutions $u \in W^{1, p}(\Omega)$, positive or not, to the problem

$$
\left\{\begin{aligned}
-\operatorname{div}\left(|\nabla u|^{p-2} \nabla u\right)+a(x)|u|^{p-2} u & =f|u|^{p^{\star}-2} u \\
u \text { is not identically zero }, u & =0 \text { on } \partial \Omega
\end{aligned}\right.
$$

where $p \in] 1, n\left[, p^{\star}=\frac{n p}{n-p}\right.$, and $a$ and $f$ are two smooth functions on $\bar{\Omega}, f$ is positive somewhere and $a$ is such that there exists some constant $C$ such that

$$
\int_{\Omega}|\nabla u|^{p}+a|u|^{p} \geq\left. C|| u\right|_{W^{1, p}(\Omega)} .
$$

Let us note that the expression $|\nabla u|^{p-2} \nabla u$ makes sense when $p>1$, as the function $\sigma$ such that $\sigma . \nabla u=|\nabla u|^{p}$ belongs to $L^{p / p^{-1}}(\Omega)$. To obtain solutions, we introduced the variational problem

$$
\lambda_{p}(\Omega, a, f)=\inf _{\left\{u \in W_{0}^{1, p}(\Omega), \int_{\Omega} f|u|^{p^{\star}}=1\right\}} \int_{\Omega}|\nabla u|^{p}+\int_{\Omega} a(x)|u|^{p}
$$

and we proved by using a method of concentration that there exists a non zero solution, as soon as

$$
\lambda_{p}(\Omega, a, f) \sup _{\{x, f(x)>0\}} f(x)^{1-\frac{p}{n}} K(n, p)^{p}<1
$$

where $K(n, p)$ denotes the inverse of the best constant for the embedding from $W^{1, p}(\Omega)$ into $L^{p^{\star}}(\Omega)$. Since the method employed in [6] cannot be applied to our problem, we shall approximate it by a problem analogous 
to (1.5), with a right hand side $f|u|^{1^{\star}-2} u$, and let $p$ tend to 1 . Of course, passing to the limit when $p \rightarrow 1$ will lead us to consider $B V(\Omega)$ in place of $W^{1,1}(\Omega)$, and to define several things, as $\sigma . \nabla u$ when $\nabla u$ is only a measure and $\sigma \in L^{\infty}(\Omega)$, and to give sense to the trace of $u$ on the boundary of $\partial \Omega$ when $u$ is only in $B V(\Omega)$. As the "limit" will be obtained by weak convergence in $B V(\Omega)$, we shall be led to overcome the lack of the weak continuity of the trace map by introducing the concept of "relaxed problem": these problems are used in the theory of minimal surfaces and plasticity, and with a slightly different meaning, in the theory of weakly harmonic functions. Here the relaxed problem is defined as:

$$
\inf _{\left\{u \in B V(\Omega), \int_{\Omega} f|u|^{\star}=1\right\}}\left\{\int_{\Omega}|\nabla u|+\int_{\partial \Omega}|u|+\int_{\Omega} a|u|\right\}
$$

and we shall see later that it has the same infimum as (1.4).

As an illustration of what may occur, let us consider the problem of the best constant for the Sobolev embedding of $W^{1,1}(\Omega)$ into $L^{1^{\star}}(\Omega)$, which corresponds to the case where $a=0$ and $f=1$. Let us define

$$
\lambda(\Omega, 0,1)=\inf _{\left\{u \in W_{0}^{1,1}(\Omega), \int_{\Omega}|u|^{\star}=1\right\}} \int_{\Omega}|\nabla u| .
$$

We have

$$
(\lambda(\Omega, 0,1))^{-1}=K(n, 1, \Omega)=\sup _{\left\{u \in W_{0}^{1,1}(\Omega)\right\}}\left\{\frac{\left(\int_{\Omega}|u|^{1^{\star}}\right)^{\frac{1}{1 \star}}}{\int_{\Omega}|\nabla u|}\right\} .
$$

This problem has been studied by many authors (see [1,14], also [4] and others...). They proved that for any open set $\Omega$ of $\mathbf{R}^{n}, K(n, 1, \Omega)=K\left(n, 1, \mathbf{R}^{n}\right)(=K(n, 1)$ for simplicity in the sequel) and that the infimum is never achieved on $W^{1,1}(\Omega)$, but in some sense, it is achieved on $B V(\Omega)$, since every characteristic function of ball whose closure is included in $\Omega$ realizes this supremum. As a consequence, $\lambda(\Omega, 0,1)=n^{1-1 / n}\left|S_{n-1}\right|^{\frac{1}{n}}=K(n, 1)^{-1}$. To be more correct, the characteristic functions of balls are solutions of (1.8) defined as:

$$
\inf _{\left\{u \in B V(\Omega), u=0 \text { on } \partial \Omega, \int_{\Omega}|u|^{1^{\star}}=1\right\}}\left\{\int_{\Omega}|\nabla u|\right\}
$$

and also of its relaxed form:

$$
\inf _{\left\{u \in B V(\Omega), \int_{\Omega}|u|^{1^{\star}}=1\right\}}\left\{\int_{\Omega}|\nabla u|+\int_{\partial \Omega}|u|\right\} .
$$

This infimum has also the value $K(n, 1)^{-1}$, as one can see by using approximation of functions in $B V(\Omega)$ by regular functions for a topology related to the narrow convergence of bounded measures, which will be precised later.

In order to give sense to equations (1.2), when $u$ is only in $B V(\Omega)$, we need to define $\sigma . \nabla u$ when $\sigma \in$ $L^{\infty}\left(\Omega, \mathbf{R}^{n}\right)$ and $u \in B V(\Omega)$ (this is possible as soon as $\operatorname{div} \sigma \in L^{n}(\Omega)$ ):

Suppose that $\varphi \in \mathcal{D}(\Omega)$, that $u \in B V(\Omega), \sigma \in L^{\infty}(\Omega)$ and $\operatorname{div} \sigma \in L^{n}(\Omega)$, and define

$$
\langle\sigma . \nabla u, \varphi\rangle=-\int_{\Omega} \operatorname{div} \sigma u \varphi-\int_{\Omega} \sigma . \nabla \varphi u .
$$

Then

$$
|\langle\sigma . \nabla u, \varphi\rangle| \leq|\sigma|_{\infty}\langle|\nabla u|,|\varphi|\rangle \cdot
$$

In particular, $\sigma . \nabla u$ is a bounded measure on $\Omega$ which is absolutely continuous with respect to $|\nabla u|$.

We now give the results of this paper: for simplicity in the sequel we drop the terms $\Omega, a$, and $f$ in the definition of $\lambda(\Omega, a, f)$. Moreover, we assume that $\Omega$ is invariant under some subgroup $G$ of the orthogonal 
group on $\mathbf{R}^{n}, O\left(\mathbf{R}^{n}\right)$, as well as $a$ and $f$. Since every $G$-invariant function is also $\bar{G}$-invariant one can assume that $G$ is compact. We denote by $O_{G}(x)$ the orbit of $x$ under $G$, and we are looking for a solution of (1.2) which is $G$-invariant. Of course, whenever $\Omega$ does not present any symmetries, one must take $G=I d$ and $O_{G}(x)=\{x\}$. The result is the following:

Theorem 1. Assume that $\Omega$ is invariant under some group $G$ of isometries of $\mathbf{R}^{n}$, as well as a and $f$, that $f$ is positive somewhere, that a satisfies (1.1). Define

$$
\lambda(G)=\inf _{\left\{u \in W_{0}^{1,1}(\Omega), u \text { is } G \text {-invariant }, \int_{\Omega} f|u|^{1^{\star}}=1\right\}}\left\{\int_{\Omega}|\nabla u|+\int_{\Omega} a|u|\right\} .
$$

Then, if for all $x \in \bar{\Omega}, \lambda(G) f(x)^{\frac{n-1}{n}} K(n, 1)\left(\operatorname{Card}_{G}(x)\right)^{\frac{-1}{n}}<1$, there exists a solution to the relaxed problem:

$$
\lambda(G)=\inf _{\left\{u \in B V(\Omega), u \text { is } G-\text { invariant } \int_{\Omega} f|u|^{1^{\star}}=1\right\}}\left\{\int_{\Omega}|\nabla u|+\int_{\partial \Omega}|u|+\int_{\Omega} a|u|\right\} .
$$

Moreover this solution verifies the P.D.E.

$$
\left\{\begin{array}{c}
-\operatorname{div} \sigma+a(x) \text { sign } u=\lambda(G) f|u|^{1^{\star}-2} u \\
\left.\sigma \in L^{\infty}\left(\Omega, \mathbf{R}^{n}\right)\right), \sigma \cdot \nabla u=|\nabla u| \\
\sigma \cdot n(u)=-|u| \text { on } \partial \Omega \\
u \neq 0, u \text { is } G-\text { invariant. }
\end{array}\right.
$$

Finally, we have another theorem which gives sufficient conditions when $\Omega$ is invariant under some symmetries, to get nodal solutions (i.e. which change sign): we assume now that $G$ is some subgroup of $O\left(\mathbf{R}^{n}\right)$ and $s$ is some involution, such that $G$ and $s$ commute weakly: $\left(s\left(O_{G}(x)\right)=O_{G}(s(x))\right.$ for all $x \in \Omega$. We then have the following result.

Theorem 2. Suppose that $G$ is some subgroup of $O\left(\mathbf{R}^{n}\right)$, that $s$ is an involution such that $s$ and $G$ commute weakly. Let $H=[G, s]$ be the group generated by $G$ and $s$. Assume that $\Omega$, a and $f$ are invariant under $H$, that $a$ and $f$ are continuous, $f$ being positive somewhere and a satisfying (1.1). Define

$$
\lambda_{G}^{s}=\inf _{\left\{u \in W_{0}^{1,1}(\Omega), u \text { is } \mathrm{G}-\text { invariant } u \circ s=-u\right\}}\left\{\int_{\Omega}|\nabla u|+\int_{\Omega} a|u|\right\}
$$

then, if for all $x \in \bar{\Omega}$

$$
\left(\operatorname{card} O_{H}(x)\right)^{\frac{-1}{n}} f(x)^{\frac{n-1}{n}} \lambda_{G}^{s} K(n, 1)<1,
$$

then there exists $u \in B V(\Omega)$ which is a solution of the relaxed problem

$$
\inf _{\{u \in B V(\Omega), u \text { is } \mathrm{G}-\text { invariant }, u \circ s=-u\}}\left\{\int_{\Omega}|\nabla u|+\int_{\partial \Omega}|u|+\int_{\Omega} a|u|\right\} .
$$

Moreover $u$ solves the partial differential equation:

$$
\left\{\begin{array}{c}
-\operatorname{div} \sigma+a(x) \operatorname{sign} u=\lambda_{G}^{s} f|u|^{1^{\star}-2} u \\
\sigma \in L^{\infty}\left(\Omega, \mathbf{R}^{n}\right), \sigma . \nabla u=|\nabla u| . \\
\sigma \cdot n(u)=-|u| \text { on } \partial \Omega \\
u \text { is } G-\text { invariant, } u \circ s=-u .
\end{array}\right.
$$

The plan of this paper is as follows: in the first section, we give an existence theorem for (1.11) and (1.12), by using both the theory of concentration compactness and the method employed in [6]. The solution belongs 
to $B V(\Omega)$, satisfies (1.11) but not necessarily the condition $u=0$ on $\partial \Omega$. Let us point out that we have no regularity result on $u$. This will probably be a difficult task, which will be the object of a future work.

In the second section, we use test functions to prove that the sufficient condition proposed previously can be traduced by very simple conditions on $a$ and $f$ and their derivatives on a point $x_{0} \in \Omega$ where $f$ achieves its maximum and then to conclude to the existence of a solution.

In the third section, we give specific examples.

\section{EXISTENCE'S THEOREMS}

\subsection{Preliminary results on BV functions}

Let us recall the definition of the space

$$
B V(\Omega)=\left\{u \in L^{1}(\Omega), \nabla u \in M^{1}(\Omega)\right\}
$$

where $\Omega$ is an open set of $\mathbf{R}^{n}, M^{1}(\Omega)$ denotes the space of bounded measures on $\Omega$, i.e. the dual space of $\mathcal{C}_{b}(\Omega)$, the space of continuous bounded functions on $\Omega$. Of course, endowed with the norm

$$
\|u\|=|u|_{L^{1}(\Omega)}+|\nabla u|_{M^{1}(\Omega)}
$$

$B V(\Omega)$ is a Banach space. The main results about $B V(\Omega)$ that we need to know here are the following: (the interested reader can consult Giusti [8] for more complete results on BV functions.)

- $B V(\Omega)$ is continuously embedded in $L^{q}(\Omega)$, for $q \leq \frac{n}{n-1}=1^{\star}$, and these embeddings are compact for $q<\frac{n}{n-1}=1^{\star}$.

Other topologies than the topology of the norm introduced above, are of importance:

- The weak topology, which can be defined as follows:

if

$$
u_{n} \rightarrow u \text { in } B V(\Omega) \text { weakly }
$$

$$
\left\{\begin{aligned}
u_{n} & \rightarrow u \text { in } L^{1}(\Omega) \\
\nabla u_{n} & \rightarrow \nabla u \text { in } M^{1}(\Omega) \text { vaguely. }
\end{aligned}\right.
$$

- The narrow topology, defined by

$$
\left\{\begin{aligned}
u_{n} & \rightarrow u \text { in } L^{1}(\Omega) \\
\nabla u_{n} & \rightarrow \nabla u \text { in } M^{1}(\Omega) \text { weakly } \\
\int_{\Omega}\left|\nabla u_{n}\right| & \rightarrow \int_{\Omega}|\nabla u| .
\end{aligned}\right.
$$

Let us note that the third condition in (1.16), jointed to the first one, implies the second one and also the vague convergence of $\left|\nabla u_{n}\right|$ towards $|\nabla u|$.

- There exists a map from $B V(\Omega)$ into $L^{1}(\partial \Omega)$, which is linear and continuous for the strong topology and coincides with the restriction on the boundary for functions in $B V(\Omega) \cap \mathcal{C}(\bar{\Omega})$. This map is not continuous for the weak topology, but it is continuous for the narrow topology. (cf. [5])

- There exists some density result of $W^{1,1}(\Omega) \cap C^{\infty}(\Omega)$ into $B V(\Omega)$ for the narrow topology defined above: For $u \in B V(\Omega)$, there exists $u_{m} \in W^{1,1}(\Omega) \cap C^{\infty}(\Omega)$, such that

$$
\left\{\begin{array}{c}
u_{m} \rightarrow u \in L^{q}(\Omega), \forall q \leq \frac{n}{n-1} \\
\int_{\Omega}\left|\nabla u_{m}\right| \rightarrow \int_{\Omega}|\nabla u| \\
u_{m}=u \text { on } \partial \Omega .
\end{array}\right.
$$


Another density result is the following:

Suppose that $\Omega$ is an open set in $\mathbf{R}^{n}$ whose boundary is piecewise $\mathcal{C}^{1}$, and that $u \in B V(\Omega)$. Then, there exists a sequence $u_{n} \in \mathcal{C}_{c}^{1}(\Omega)$, such that

$$
\left\{\begin{aligned}
u_{n} & \rightarrow u \text { in } B V(\Omega) \text { weakly } \\
\int_{\Omega}\left|\nabla u_{n}\right| & \rightarrow \int_{\Omega}|\nabla u|+\int_{\partial \Omega}|u| .
\end{aligned}\right.
$$

As a corollary of this result, one obtains that the inequality in (1.1) can be extended to functions of $B V(\Omega)$ in the following manner: There exists some constant $C>0$ such that for all $u \in B V(\Omega)$

$$
\int_{\Omega}|\nabla u|+\int_{\partial \Omega}|u|+\int_{\Omega} a|u| \geq C|| u \|_{B V(\Omega)}
$$

The proof of this approximation result is postponed in the fifth step in part II, since it is a key ingredient to prove that

$$
\inf (1.11)=\inf (1.10)
$$

We shall also need a generalization of Green's formula, which gives some sense to $\sigma . \nabla u$ when $\sigma \in L^{\infty}(\Omega)$ and $u \in B V(\Omega)$, as soon as $\operatorname{div} \sigma \in L^{n}(\Omega)$. (This result has already been announced in the introduction.)

Proposition 1. Suppose that $\sigma \in L^{\infty}\left(\Omega, \mathbf{R}^{n}\right)$, div $\sigma \in L^{n}(\Omega)$, and $u \in B V(\Omega)$. Then there exists a distribution, denoted as $\sigma . \nabla u$, which is defined as follows:

$$
\langle\sigma \cdot \nabla u, \varphi\rangle=-\int_{\Omega} u(\operatorname{div}) \sigma \varphi-\int_{\Omega} u \sigma \cdot \nabla \varphi
$$

for $\varphi \in \mathcal{C}^{\infty}(\Omega)$.

The distribution $\sigma . \nabla u$ is a bounded measure which satisfies:

$$
|\sigma . \nabla u| \leq|\sigma|_{\infty}|\nabla u| .
$$

In addition, if $\varphi \in \mathcal{C}(\bar{\Omega}) \cap \mathcal{C}^{1}(\Omega)$, the following Green's Formula holds

$$
\langle\nabla u \cdot \sigma, \varphi\rangle=-\int_{\Omega} u(\operatorname{div} \sigma) \varphi-\int_{\Omega} \sigma \cdot \nabla \varphi u+\int_{\partial \Omega} \sigma \cdot \vec{n} u \varphi .
$$

- Suppose that $U \in B V\left(\mathbf{R}^{N}\right)$, and define for $u \in B V(\Omega)$ the function $\tilde{u}$ as

$$
\tilde{u}=\left\{\begin{array}{l}
u \text { in } \Omega \\
U \text { in } \mathbf{R}^{N}-\bar{\Omega} .
\end{array}\right.
$$

Then $\tilde{u} \in B V\left(\mathbf{R}^{N}\right)$ and

$$
\nabla \tilde{u}=\nabla u \chi_{\Omega}+\nabla U \chi_{\left\{\mathbf{R}^{N}-\bar{\Omega}\right\}}+(U-u)_{\mid \Omega} \delta_{\partial \Omega}
$$

where $U_{\mid \Omega}$ and $u_{\mid \Omega}$ denote the trace of $U$ and $u$ on $\partial \Omega$. (cf. $\left.[8,15,16]\right), \delta_{\partial \Omega}$ denotes the uniform Dirac measure on $\partial \Omega$ and $\vec{n}$ is the unit outer normal to $\partial \Omega$. Moreover, one can define the measure $\sigma \cdot \nabla \tilde{u}$ on $\bar{\Omega}$ by the formula

$$
(\sigma \cdot \nabla \tilde{u})=(\sigma \cdot \nabla u) \chi_{\Omega}+\sigma \cdot \vec{n}(U-u) \delta_{\partial \Omega}
$$

and $\sigma \cdot \nabla \tilde{u}$ is absolutely continuous with respect to $|\nabla \tilde{u}|$, with the inequality

$$
|\sigma \cdot \nabla \tilde{u}| \leq|\sigma|_{\infty}|\nabla \tilde{u}| \cdot
$$

We end this section by enouncing a lemma which is classical in the theory of $B V$-functions: 
Lemma 1. Assume that $\Omega$ is an open set of $\mathbf{R}^{n}, n \geq 2$, and that $u \in B V(\Omega)$. Then, if $x_{0} \in \Omega,|\nabla u|\left(\left\{x_{0}\right\}\right)=0$.

Remark. Lemma 1 is a mere consequence of a stronger result which says that $|\nabla u|(A)=0$ as soon as the (n-1)-Hausdorff measure of A is zero.

A proof of this stronger result can be found in [8]. We give here an elementary one.

Proof of Lemma 1. We must prove that

$$
\lim _{r \rightarrow 0} \int_{C\left(x_{0}, r\right)}|\nabla u|=0
$$

where $C\left(x_{0}, r\right)$ denotes the open n-cube $] x_{0}-r, x_{0}+r{ }^{n}$. We write

$$
|\nabla u|\left(C\left(x_{0}, r\right)\right)=\int_{\left\{C\left(x_{0}, r\right) \cap\left\{x_{n}>0\right\}\right\}}|\nabla u|+\int_{\left\{C\left(x_{0}, r\right) \cap\left\{x_{n}<0\right\}\right\}}|\nabla u|+\int_{\left\{C\left(x_{0}, r\right) \cap\left\{x_{n}=0\right\}\right\}}|[u]|\left(x^{\prime}, 0\right) d x^{\prime}
$$

where $[u]$ denotes the jump of the trace of $u$ on the hyperplan $x_{n}=0$. Since it belongs to $L^{1}\left(\Omega \cap\left\{x_{n}=0\right\}\right)$, one has

$$
\lim _{r \rightarrow 0} \int_{C\left(x_{0}, r\right) \cap\left\{x_{n}=0\right\}}|[u]|\left(x^{\prime}, 0\right) d x^{\prime}=0 .
$$

On another hand, $|\nabla u|$ being a bounded measure on $\Omega$

$$
\varlimsup_{r \rightarrow 0} \int_{C\left(x_{0}, r\right) \cap\left\{x_{n}>0\right\}}|\nabla u| \leq \varlimsup_{r \rightarrow 0} \int_{\Omega \cap\left\{r>x_{n}>0\right\}}|\nabla u|=0
$$

and

$$
\varlimsup_{r \rightarrow 0} \int_{C\left(x_{0}, r\right) \cap\left\{-r<x_{n}<0\right\}}|\nabla u| \leq \varlimsup_{r \rightarrow 0} \int_{C\left(x_{0}, r\right) \cap\left\{x_{n}<0\right\}}|\nabla u|=0 .
$$

This yields the desired conclusion.

\subsection{Proof of Theorem 1}

A first step when one tries to prove Theorem 1 consists in approximating (1.11) with the following minimization problem

$$
\lambda_{\epsilon}(G)=\inf _{\left\{u \in W_{0}^{1,1+\epsilon}(\Omega), u \circ \tau=u, \forall \tau \in G, \int_{\Omega} f(x)|u|^{1^{\star}}=1\right\}}\left\{\int_{\Omega}|\nabla u|^{1+\epsilon}+\int_{\Omega} a(x)|u|^{1+\epsilon}\right\},
$$

where $\epsilon$ is some positif parameter. This problem can be solved by classical methods in the calculus of variations, since $1^{\star}$ is strictly less than the critical power for the embedding of $W^{1,1+\epsilon}(\Omega)$ into $L^{q}(\Omega)$. Let us note that a solution of (1.20) verifies: $\forall \varphi \in \mathcal{D}(\Omega, \mathbf{R})$ such that $\varphi \circ \tau=\varphi, \forall \tau \in G$,

$$
-\int_{\Omega} \operatorname{div} \sigma_{\epsilon} \varphi+\int_{\Omega} a\left|u_{\epsilon}\right|^{\epsilon-1} u_{\epsilon} \varphi=\lambda_{\epsilon}(G) \int_{\Omega} f\left|u_{\epsilon}\right|^{1^{\star}-2} u_{\epsilon} \varphi .
$$

In order to prove that equation (1.21) may be extended to every $\varphi \in \mathcal{D}(\Omega)$, suppose by contradiction that there exists $\varphi \in \mathcal{D}(\Omega)$ such that

$$
-\int_{\Omega} \operatorname{div} \sigma_{\epsilon} \varphi+\int_{\Omega} a\left|u_{\epsilon}\right|^{\epsilon-1} u_{\epsilon} \varphi-\lambda_{\epsilon}(G) \int_{\Omega} f\left|u_{\epsilon}\right|^{1^{\star}-2} u_{\epsilon} \varphi=\alpha>0 .
$$

Then for all $\tau \in G$,

$$
-\int_{\Omega} \operatorname{div} \sigma_{\epsilon} \varphi \circ \tau+\int_{\Omega} a\left|u_{\epsilon}\right|^{\epsilon-1} u_{\epsilon} \varphi \circ \tau-\lambda_{\epsilon}(G) \int_{\Omega} f\left|u_{\epsilon}\right|^{1^{\star}-2} u_{\epsilon} \varphi \circ \tau=\alpha .
$$


Using the existence of a Haar measure $d \mu$ on $G$, the $G$-invariance of $\Omega, a$ and $f$, and integrating this with respect to the Haar measure $d \mu$ on $G$ one obtains

$$
-\int_{\Omega} \operatorname{div} \sigma_{\epsilon} \varphi_{G}+\int_{\Omega} a\left|u_{\epsilon}\right|^{\epsilon-1} u_{\epsilon} \varphi_{G}-\lambda_{\epsilon}(G) \int_{\Omega} f\left|u_{\epsilon}\right|^{1^{\star}-2} u_{\epsilon} \varphi_{G}=\alpha
$$

where $\varphi_{G}=\frac{\int_{G} \varphi \circ \tau d \mu(\tau)}{\int_{G} d \mu(\tau)}$. Since $\varphi_{G}$ is $G$-invariant and compactly supported in $\Omega$, one obtains a contradiction with (1.21).

As a consequence, $u_{\epsilon}$ is a non trivial solution of

$$
\left\{\begin{aligned}
-\operatorname{div} \sigma_{\epsilon}+a(x)\left|u_{\epsilon}\right|^{\epsilon-1} u_{\epsilon} & =\lambda_{\epsilon} f(x)\left|u_{\epsilon}\right|^{1^{\star}-2} u_{\epsilon} \text { in } \Omega \\
\sigma_{\epsilon} & =\left|\nabla u_{\epsilon}\right|^{\epsilon-1} \nabla u_{\epsilon} \\
u_{\epsilon} & \in W_{0}^{1,1+\epsilon}(\Omega) .
\end{aligned}\right.
$$

\section{Second step}

\section{Proposition 2.}

$$
\varlimsup_{\epsilon \rightarrow 0} \lambda_{\epsilon}(G)=\bar{\lambda} \leq \lambda(G)
$$

Proof. Let $I_{\epsilon}(v)=\int_{\Omega}|\nabla v|^{1+\varepsilon}+\int_{\Omega} a(x)|v|^{1+\varepsilon}$, and let $\delta>0$ be given and $v \in \mathcal{C}_{c}^{\infty}(\Omega)$ be $G$-invariant, such that $\int_{\Omega} f|v|^{1 \star}=1$ and $\int_{\Omega}|\nabla v|+\int_{\Omega} a(x)|v| \leq \lambda+\delta$. For $\epsilon$ small enough, $\left|I_{\epsilon}(v)-I_{0}(v)\right|<\delta$, hence

$$
\varlimsup_{\epsilon \rightarrow 0} \lambda_{\epsilon}(G) \leq \lambda(G)+\delta
$$

$\delta$ being arbitrary, we get $\varlimsup_{\epsilon \rightarrow 0} \lambda_{\epsilon} \leq \lambda$.

Let now $u_{\epsilon}$ be a solution of (1.20). Then, it is bounded in $W^{1,1+\epsilon}(\Omega)$. Therefore, we may extract from it a subsequence, still denoted $u_{\epsilon}$, such that

$$
\begin{gathered}
u_{\epsilon} \rightarrow u \text { in } L^{p}(\Omega), \text { for all } p<1^{\star} \\
\nabla u_{\epsilon} \rightarrow \nabla u \text { in } M^{1}(\Omega) \text { weakly } \\
u_{\epsilon} \rightarrow u \text { in } L^{1^{\star}}(\Omega) \text { weakly }
\end{gathered}
$$

(the second assertion is a consequence of Hölder's inequality

$$
\left.\int\left|\nabla u_{\epsilon}\right| \leq\left(\int\left|\nabla u_{\epsilon}\right|^{1+\epsilon}\right)^{\frac{1}{1+\epsilon}}(\operatorname{mes}(\Omega))^{\frac{\epsilon}{1+\epsilon}}\right) .
$$

We need now to recall a result of concentration compactness, which is a consequence of Lions' concentration compactness theory [11]:

Proposition 3. 1) Suppose that $\Omega$ is an open bounded set in $\mathbf{R}^{N}$ and that $u_{\epsilon}$ is bounded in $W^{1,1+\epsilon}(\Omega)$, then if $u_{\epsilon} \rightarrow u \in B V(\Omega)$ weakly, there exists two nonnegative bounded measures on $\Omega, \nu$ and $\mu$, a numerable set $\left(x_{i}\right)_{i \in \mathbf{N}} \in \bar{\Omega}$, and some numbers $\nu_{i} \geq 0, \mu_{i} \geq 0$, such that

$$
\begin{gathered}
\left|\nabla u_{\epsilon}\right|^{1+\epsilon} \rightarrow \mu \geq|\nabla u|+\sum_{i} \mu_{i} \delta_{x_{i}} \text { in } M^{1}(\Omega) \text { weakly } \\
\left|u_{\epsilon}\right|^{1^{\star}} \rightarrow|u|^{1^{\star}}+\sum_{i} \nu_{i} \delta_{x_{i}} \text { in } M^{1}(\Omega) \text { weakly }
\end{gathered}
$$

where $\delta_{x_{i}}$ denotes the Dirac mass on $x_{i}$. Moreover

$$
\nu_{i} \leq K(n, 1) \mu_{i}^{\frac{1}{1^{*}}}
$$


2) If $u_{\epsilon}$ is bounded in $W^{1,1+\epsilon}\left(\mathbf{R}^{n}\right)$ and if the functions $u_{\epsilon}$ have their support included in some fixed compact set $K$ the conclusion is the same with $\mu \in M^{1}\left(\mathbf{R}^{n}\right), \nu \in M^{1}\left(\mathbf{R}^{n}\right)$ with support in $K$, and the points $x_{i}$ belong to $K$.

Remark. If $u_{\epsilon}$ is $G$-invariant for all $\epsilon$, so are the measures $\mu$ and $\nu$, and so is the set $\left\{x_{i}\right\}$.

Proof. It suffices to prove that $\left|\nabla u_{\epsilon}\right|$ is bounded in $W^{1,1}(\Omega)$ and has a limit (up to subsequences) less than the limit of $\left|\nabla u_{\epsilon}\right|^{1+\epsilon}$, and to use the results of Lions ([11], see also [5]). For that aim, let $\varphi \in \mathcal{D}(\Omega), \varphi \geq 0$. By Hölder's inequality we have:

$$
\int\left|\nabla u_{\epsilon}\right| \varphi \leq\left(\int\left|\nabla u_{\epsilon}\right|^{1+\epsilon} \varphi\right)^{\frac{1}{1+\epsilon}}\left(\int(\varphi)\right)^{\frac{\epsilon}{1+\epsilon}}
$$

Since the last integral on the right tends to 1 , we obtain the conclusion.

Third step: We obtain $\sigma=" \frac{\nabla u}{|\nabla u|} "$ as the weak limit of $\sigma_{\epsilon}=\left|\nabla u_{\epsilon}\right|^{\epsilon-1} \nabla u_{\epsilon}$.

Let $\sigma_{\epsilon}=\left|\nabla u_{\epsilon}\right|^{\epsilon-1} \nabla u_{\epsilon}$. Then $\sigma_{\epsilon}$ belongs to $L^{\frac{1+\epsilon}{\epsilon}}(\Omega)$. Let us prove that $\sigma_{\epsilon} \rightarrow \sigma \in L^{q}(\Omega)$ weakly for some $\sigma \in L^{q}(\Omega)$, for a subsequence, and for all $q<\infty$. For that aim, let $\epsilon<\epsilon^{\prime}$, then $\frac{1+\epsilon^{\prime}}{\epsilon^{\prime}}<\frac{1+\epsilon}{\epsilon}$, and by Hölder's inequality,

$$
\int_{\Omega}\left|\sigma_{\epsilon}\right|^{\frac{1+\epsilon^{\prime}}{\epsilon^{\prime}}} \leq\left(\int_{\Omega}\left|\sigma_{\epsilon}\right|^{\frac{1+\epsilon}{\epsilon}}\right)^{\frac{\epsilon\left(1+\epsilon^{\prime}\right)}{\epsilon^{\prime}(1+\epsilon)}}(\operatorname{mes}(\Omega))^{1-\frac{\epsilon\left(1+\epsilon^{\prime}\right)}{\epsilon^{\prime}(1+\epsilon)}}
$$

since $\left.\frac{\epsilon\left(1+\epsilon^{\prime}\right)}{\epsilon^{\prime}(1+\epsilon)} \in\right] 0,1\left[\right.$. By passing to the limit when $\epsilon$ goes to 0 , one obtains that $\sigma_{\epsilon}$ tends to $\sigma$ weakly in $L^{q}(\Omega), \forall q<\infty$. Moreover, for all $\epsilon^{\prime}>0$, we have

$$
|\sigma|_{L^{1+\frac{1}{\epsilon^{\prime}}}} \leq \text { mes } \Omega \text {. }
$$

We need to prove that $|\sigma|_{\infty} \leq 1$. For that aim, let $\eta$ be in $\mathcal{D}\left(\Omega, \mathbf{R}^{n}\right)$. Then

$$
\begin{aligned}
\left|\int_{\Omega} \sigma \cdot \eta\right| & \leq \underline{\lim }_{\epsilon \rightarrow 0}\left|\int_{\Omega} \sigma_{\epsilon} \cdot \eta\right| \leq \varliminf_{\epsilon \rightarrow 0} \int_{\Omega}\left|\nabla u_{\epsilon}\right|^{\epsilon}|\eta| \leq \varliminf_{\epsilon \rightarrow 0}\left(\int_{\Omega}\left|\nabla u_{\epsilon}\right|^{1+\epsilon}\right)^{\frac{\epsilon}{1+\epsilon}}\left(\int_{\Omega} \eta^{1+\epsilon}\right)^{\frac{1}{1+\epsilon}} \\
& \leq \varliminf_{\epsilon \rightarrow 0}(C)^{\frac{\epsilon}{1+\epsilon}}\left(\int_{\Omega} \eta^{1+\epsilon}\right)^{\frac{1}{1+\epsilon}} \leq \int_{\Omega}|\eta| .
\end{aligned}
$$

This implies that $|\sigma| \leq 1$. On the other hand, by passing to the limit in (1.23), one gets:

$$
-\operatorname{div} \sigma+a \operatorname{sign} u=\bar{\lambda} f|u|^{1^{\star}-2} u
$$

and $u$ is $G$-invariant, as the limit almost everywhere of $G$-invariant functions.

Fourth step: Extension of $u_{\epsilon}$ outside $\Omega$ and convergence towards a solution of (1.11).

Let $\tilde{u}_{\epsilon}$ be the extension of $u_{\epsilon}$ by 0 in $\mathbf{R}^{n}-\bar{\Omega}$. Then $\tilde{u}_{\epsilon} \in W^{1,1+\epsilon}\left(\mathbf{R}^{n}\right)$, since $u_{\epsilon}=0$ on $\partial \Omega$, and $\left(\tilde{u}_{\epsilon}\right)$ is bounded in $W^{1,1}\left(\mathbf{R}^{n}\right)$. Then, one may extract from it a subsequence, still denoted $\left(\tilde{u}_{\epsilon}\right)$ such that

$$
\begin{aligned}
& \tilde{u}_{\epsilon} \rightarrow v \text { in } L^{k}\left(\mathbf{R}^{n}\right), \forall k<\frac{n}{n-1} \\
& \tilde{u}_{\epsilon} \rightarrow v \text { in } L^{1^{\star}}\left(\mathbf{R}^{n}\right)
\end{aligned}
$$

with $v=0$ outside of $\Omega$. We denote by $u$ the restriction of $v$ to $\Omega$. In addition:

$$
\begin{aligned}
\nabla \tilde{u}_{\epsilon} & \rightarrow \nabla v \text { in } M^{1}\left(\mathbf{R}^{n}\right) \\
\sigma_{\epsilon} & =\left|\nabla u_{\epsilon}\right|^{\epsilon-1} \nabla u_{\epsilon} \rightarrow \sigma \text { in } L^{q}(\Omega) \forall q<\infty .
\end{aligned}
$$


Moreover there exists two nonnegative measures $\mu$ and $\nu$, with support in $\bar{\Omega}$, a numerable set $\left\{x_{i}\right\}$ in $\bar{\Omega}$, and some reals $\mu_{i}$ and $\nu_{i}$, such that

$$
\begin{aligned}
\left|\nabla \tilde{u}_{\epsilon}\right|^{1+\epsilon}-\mu & \geq|\nabla v|+\sum_{i} \mu_{i} \delta_{x_{i}} \\
\left|\tilde{u}_{\epsilon}\right|^{1^{\star}}-\nu & =|u|^{1^{\star}}+\sum_{i} \nu_{i} \delta_{x_{i}} .
\end{aligned}
$$

Multiplying (1.23) by $\tilde{u}_{\epsilon} \varphi$ where $\varphi \in \mathcal{D}\left(\mathbf{R}^{n}\right)$, and integrating by parts, one obtains:

$$
\int_{\bar{\Omega}} \sigma_{\epsilon} \cdot \nabla\left(\tilde{u}_{\epsilon} \varphi\right)+\int_{\Omega} a(x)\left|\tilde{u}_{\epsilon}\right|^{1+\epsilon} \varphi=\lambda_{\epsilon}(G) \int_{\Omega} f\left|\tilde{u}_{\epsilon}\right|^{1^{\star}} \varphi
$$

or equivalently

$$
\int_{\mathbf{R}^{n}}\left|\nabla\left(\tilde{u}_{\epsilon}\right)\right|^{1+\epsilon} \varphi+\int_{\mathbf{R}^{n}} \sigma_{\epsilon} \tilde{u}_{\epsilon} \nabla \varphi+\int_{\mathbf{R}^{n}} a(x)\left|\tilde{u}_{\epsilon}\right|^{1+\epsilon} \varphi=\lambda_{\epsilon}(G) \int_{\mathbf{R}^{n}} f\left|\tilde{u}_{\epsilon}\right|^{1^{\star}} \varphi
$$

By assuming that $\epsilon<\frac{1}{n-1}, \sigma_{\epsilon}$ tends weakly towards $\sigma$ in $L^{n+\alpha}(\Omega)$ for some $\alpha>0$, and then, since $\tilde{u}_{\epsilon}$ tends strongly towards $v$ in every $L^{q}, q<\frac{n}{n-1}$, one obtains that $\int_{\mathbf{R}^{n}} \sigma_{\epsilon} \tilde{u}_{\epsilon} \cdot \nabla \varphi$ tends to $\int_{\mathbf{R}^{n}} \sigma v \cdot \nabla \varphi$. By passing to the limit in the last equation above, one obtains

$$
\langle\mu, \varphi\rangle+\int_{\Omega} \sigma u \nabla \varphi+\int_{\Omega} a|u| \varphi=\bar{\lambda}\left(\int_{\Omega} f|v|^{1^{\star}} \varphi+\sum_{i} \nu_{i} \varphi\left(x_{i}\right) f\left(x_{i}\right)\right)=\bar{\lambda}\langle\nu, f \varphi\rangle .
$$

Using generalised Green's Formula in Proposition 1 and (1.27) one obtains that

$$
\begin{aligned}
\int_{\Omega} \sigma u \cdot \nabla \varphi & =-\int_{\Omega} \operatorname{div} \sigma u \varphi-\int_{\Omega} \sigma \cdot \nabla u \varphi+\int_{\partial \Omega} \sigma \cdot \vec{n}(u) \varphi \\
& =\bar{\lambda} \int_{\Omega} f|v|^{1^{\star}} \varphi-\int_{\Omega} a(x)|u| \varphi-\int_{\bar{\Omega}} \sigma \cdot \nabla v \varphi .
\end{aligned}
$$

Subtracting (1.29) from (1.28), one gets for $\varphi \in \mathcal{D}(\bar{\Omega})$

$$
\langle\mu, \varphi\rangle-\int_{\bar{\Omega}} \sigma . \nabla v \varphi=\bar{\lambda} \sum_{i} \nu_{i} f\left(x_{i}\right) \varphi\left(x_{i}\right)
$$

Let now $h$ be a $|\nabla v|$-measurable function and $\mu^{\perp}$ be a measure orthogonal to $|\nabla u|$, such that, according to the Radon-Nikodym decomposition, one has

$$
\mu=h|\nabla v|+\mu^{\perp}
$$

By Lemma 1 and the analogous of (1.24) in 2) of Proposition 3, one has

$$
h|\nabla v| \geq|\nabla v|
$$

and

$$
\mu^{\perp} \geq \sum_{i} \mu_{i} \delta_{x_{i}}
$$

Using (1.32) and (1.33) in equation (1.30) one gets that

$$
h|\nabla v|=\sigma . \nabla v \text { on } \bar{\Omega}
$$


and

$$
\mu^{\perp}=\sum_{i} \mu_{i} \delta_{x_{i}}=\bar{\lambda} \sum_{i} \nu_{i} f\left(x_{i}\right) \delta_{x_{i}}
$$

From (1.35), one gets that as soon as $f\left(x_{i}\right)<0$, then $\mu_{i}=\nu_{i}=0$. Using (1.34), $|\sigma| \leq 1$ and Proposition 1 , one obtains that in the sense of measures

$$
|\sigma . \nabla v| \leq|\nabla v| \text { on } \bar{\Omega}
$$

and then

$$
\sigma . \nabla v=|\nabla v|
$$

on $\bar{\Omega}$, and $h=1,|\nabla v|$-almost everywhere. Using this in equation (1.28) with $\varphi=1$ one gets that

$$
\int_{\Omega}|\nabla v|+\int_{\partial \Omega}|v|+\int_{\Omega} a|v| \leq \bar{\lambda} \int_{\Omega} f|v|^{1^{\star}}
$$

By the remark done about the 1-coercivity of $a$, we deduce that $\int_{\Omega} f|v|^{1^{\star}} \geq 0$, and replacing this in the equation

$$
1=\int_{\Omega} f|v|^{1^{\star}}+\sum_{i} \nu_{i} f\left(x_{i}\right)
$$

one obtains that $\sum_{i} \nu_{i} f\left(x_{i}\right) \in[0,1]$ for all $i$. This implies that for all $i$,

$$
\left(f\left(x_{i}\right) \nu_{i}\right) \leq \frac{1}{\operatorname{Card}_{G}\left(x_{i}\right)}
$$

Indeed, if $\operatorname{Card}_{G}\left(x_{i}\right)<\infty$, suppose by contradiction that there exists $i \in \mathbf{N}$ such that $\left(f\left(x_{i}\right) \nu_{i}\right)>\frac{1}{\operatorname{Card} O_{G}\left(x_{i}\right)}$. Then, summing over the orbit of $x_{i}$ one obtains a contradiction with

$$
1<\left(\sum_{x_{j} \notin O_{G}\left(x_{i}\right)} f\left(x_{j}\right) \nu_{j}+\sum_{x_{k} \in O_{G}\left(x_{i}\right)} f\left(x_{k}\right) \nu_{k}+\int_{\Omega} f|v|^{1^{\star}}\right) \leq \lim _{\epsilon \rightarrow 0} \int_{\Omega} f\left|u_{\epsilon}\right|^{1 \star}=1 .
$$

Using this inequality one obtains also that $\operatorname{Card}_{G}\left(x_{i}\right)=\infty$ implies that $\nu_{i}=0$. We now write, using proposition 2, the assumption $\lambda(G) f(x)^{\frac{n-1}{n}} K(n, 1) \operatorname{Card}_{G}(x)^{\frac{-1}{n}}<1$ for all $x \in \bar{\Omega}$, and (1.26):

$$
\begin{aligned}
\mu_{i} & \leq \lambda(G) f\left(x_{i}\right)^{\frac{1}{1^{\star}}} f\left(x_{i}\right)^{1-\frac{1}{1^{\star}}} \nu_{i}^{1-\frac{1}{1^{\star}}} \nu_{i}^{\frac{1}{1 \star}} \leq \lambda(G)\left(\operatorname{card} O_{G}\left(x_{i}\right)\right)^{-1+\frac{1}{1^{\star}}} f\left(x_{i}\right)^{\frac{1}{1^{\star}}} \nu_{i}^{\frac{1}{1 \star}} \\
& \leq \lambda(G)\left(\operatorname{card} O_{G}\left(x_{i}\right)\right)^{-1+\frac{1}{1^{\star}}} f\left(x_{i}\right)^{\frac{1}{1 \star}} K(n, 1) \mu_{i}<c \mu_{i}
\end{aligned}
$$

where $c \in] 0,1\left[\right.$. This implies that $\mu_{i}=0$, for all $i$ and then $\nu_{i}=0$ for all $i$. Consequently, $\lim _{\epsilon \rightarrow 0}\left|\tilde{u}_{\epsilon}\right|^{1^{\star}}=|v|^{1^{\star}}$ and $\sigma . \nabla v=|\nabla v|$, on $\Omega \cup \partial \Omega$. This condition can be splitted in the two equations

$$
\left\{\begin{aligned}
\sigma . \nabla u & =|\nabla u| \text { in } \Omega \\
\sigma \cdot \vec{n} u & =-|u| \text { on } \Omega
\end{aligned}\right.
$$

Then $u$ is a solution of (1.12), if we are able to prove that $u$ is not identically zero. In fact, since for all $i, \nu_{i}=0$, we get from the previous computations that

$$
f\left|\tilde{u}_{\epsilon}\right|^{1^{\star}} \rightarrow f|v|^{1^{\star}}
$$

vaguely on $\mathbf{R}^{n}$, and then

$$
\int_{\mathbf{R}^{n}} f\left|\tilde{u}_{\epsilon}\right|^{1^{\star}}=1 \rightarrow \int_{\mathbf{R}^{n}} f|v|^{1^{\star}}=1
$$


As a consequence, $u$ cannot be zero, and

$$
\int_{\Omega} f|u|^{1 \star}=1
$$

Moreover, the convergence of $\left|\nabla \tilde{u}_{\epsilon}\right|$ is tight on $\bar{\Omega}$, which means that

$$
\int_{\Omega}\left|\nabla u_{\epsilon}\right| \rightarrow \int_{\Omega}|\nabla u|+\int_{\partial \Omega}|u| .
$$

\section{Fifth step: $u$ is a solution of (1.11).}

Let us recall the relaxed form of (1.10), for which we shall prove the existence of a solution.

$$
\inf _{\left\{u \in B V(\Omega), u \text { is } G-\text { invariant, } \int_{\Omega} f|u|^{1^{\star}}=1\right\}}\left\{\int_{\Omega}|\nabla u|+\int_{\partial \Omega}|u|+\int_{\Omega} a(x)|u|\right\} .
$$

As we already mentioned it before, this problem makes sense, because of the embedding of $B V(\Omega)$ into $L^{\frac{n}{n-1}}(\Omega)$, and due to the existence of a trace map from $B V(\Omega)$ into $L^{1}(\partial \Omega)$. We must prove first that

$$
\inf (1.11)=\inf (1.10) \text {. }
$$

First, it is obvious that

$$
\inf (1.11) \leq \inf (1.10)
$$

For the reverse inequality, let $u$ be in $B V(\Omega)$. Since $\Omega$ is piecewise $\mathcal{C}^{1}$, there exists a covering of $\Omega$ by a relatively compact set $\Omega_{0}$ and a finite number of balls, $B\left(x_{j}, \delta\right), 1 \leq j \leq k, x_{j} \in \Omega$, such that $\Omega \cap B\left(x_{j}, \delta\right)$ is starshaped with respect to $x_{j}$, for all $j$. Let $\varphi_{j}$ be a partition of unity subordinate to this covering. We have

$$
u=\varphi_{0} u+\sum_{j=1}^{j=k} \varphi_{j} u
$$

We always denote by $v$ the extension of $u$ by zero outside $\Omega$. Let $\lambda$ be close to $1, \lambda>1$, such that the functions

$$
\left.\left(\varphi_{j} v\right)_{\lambda}(x)=\varphi_{j} v\left(x_{j}+\lambda\left(x-x_{j}\right)\right)\right\}
$$

have compact support in

$$
\Omega_{\lambda}=\left\{x \in \Omega, d(x, \partial \Omega)>d\left(x_{j}, \partial \Omega\right)\left(\frac{\lambda-1}{\lambda}\right)\right\}
$$

$\left(\Omega_{\lambda} \subset \Omega\right.$.) Moreover, it is not difficult to see that

$$
\lim _{\lambda \rightarrow 1} \int_{\mathbf{R}^{n}}\left|\nabla\left(\varphi_{j} v\right)_{\lambda}\right|=\int_{\Omega}\left|\nabla\left(\varphi_{j} v\right)\right|+\int_{\partial \Omega}\left|\varphi_{j} v\right| .
$$

Let now $\epsilon$ be less than the distance of the support of $\left(\varphi_{j} v\right)_{\lambda}$ to $\partial \Omega$. The functions $\rho_{\epsilon} \star\left(\varphi_{j} v\right)_{\lambda}$ (where $\rho_{\epsilon}$ are standard mollifiers) are $\mathcal{C}^{\infty}$ and have compact support. Moreover

Let us define

$$
\left.\lim _{\epsilon \rightarrow 0}||\left|\rho_{\epsilon} \star\left(\varphi_{j} v\right)_{\lambda}\right|\right|_{B V(\Omega)}-||\left(\varphi_{j} v\right)_{\lambda}||_{B V(\Omega)} \mid=0 .
$$

$$
u_{\epsilon, \lambda}=\rho_{\epsilon} \star\left(\varphi_{0} v\right)+\sum_{j \geq 1} \rho_{\epsilon} \star\left(\varphi_{j} v\right)
$$

for $\lambda$ close to 1 and $\epsilon$ small enough. We have

$$
\lim _{\epsilon \rightarrow 0} \int_{\Omega} f\left|u_{\epsilon, \lambda}\right|^{1^{\star}}=1
$$


and then the function

$$
v_{\epsilon, \lambda}=\frac{u_{\epsilon, \lambda}}{\left(\int f u_{\epsilon, \lambda}^{1^{\star}}\right)^{\frac{n-1}{n}}}
$$

is in $\mathcal{D}(\Omega)$ and satisfies

$$
\int_{\Omega}\left|\nabla v_{\epsilon, \lambda}\right|+\int_{\Omega} a\left|v_{\epsilon, \lambda}\right| \rightarrow \int_{\Omega}|\nabla u|+\int_{\partial \Omega}|u|+\int_{\Omega} a|u| .
$$

Finally, let $\mu$ be the Haar measure on $G$ and let

$$
v_{\epsilon, \lambda}^{G}=\frac{\int_{G} v_{\epsilon, \lambda} \circ \sigma d \mu(\tau)}{|G|} .
$$

Then

$$
\lim \int_{\Omega}\left|\nabla v_{\epsilon, \lambda}^{G}\right|+\int_{\Omega} a\left|v_{\epsilon, \lambda}^{G}\right| \rightarrow \int_{\Omega}|\nabla u|+\int_{\partial \Omega}|u|+\int_{\Omega} a|u|
$$

and $v_{\epsilon, \lambda}^{G}=0$ on $\partial \Omega$, is $G$-invariant, and verifies $\int_{\Omega} f\left|v_{\epsilon, \lambda}^{G}\right|^{1^{\star}}=1$, which yields the desired result.

We prove now that the limit $u$ in the two previous steps is a solution of (1.11). For that aim, let us recall that $\int_{\Omega} f|v|^{1^{*}}=1$. By lower semi-continuity, we get:

$$
\lambda(G)=\int_{\mathbf{R}^{n}}|\nabla v|+\int_{\Omega} a(x)|v| \leq \lim _{\epsilon \rightarrow 0} \int_{\mathbf{R}^{n}}\left|\nabla \tilde{u_{\epsilon}}\right|^{1+\epsilon}+\int_{\Omega} a(x)\left|\tilde{u}_{\epsilon}\right|^{1+\epsilon}=\lim _{\epsilon \rightarrow 0} \lambda_{\epsilon}(G) .
$$

Using the fact that

$$
\int_{\mathbf{R}^{n}}|\nabla v|=\int_{\Omega \cup \partial \Omega}|\nabla v|=\int_{\Omega}|\nabla u|+\int_{\partial \Omega}|u|,
$$

one obtains that $u$ is a solution of the relaxed problem.

\subsection{Existence of nonnegative solutions}

We consider the same problem as previously, with the additional assumption that $u$ is nonnegative. So we are looking for a non trivial solution of

$$
\left\{\begin{array}{c}
-\operatorname{div} \sigma+a \operatorname{sign}^{+} u=\lambda(G) f u^{1^{\star}-1} \\
\sigma \cdot \nabla u=|\nabla u| \\
u \text { is nonnegative and } G-\text { invariant } \\
\sigma \in L^{\infty}\left(\Omega, \mathbf{R}^{n}\right), \sigma \cdot n(-u)=u \text { on } \partial \Omega
\end{array}\right.
$$

where $\lambda(G)$ has been defined in the previous section and $\operatorname{sign}^{+} u$ has been defined in the introduction.

As in the previous section, we shall find $u$ as a nonnegative solution to the relaxed problem (1.11). To solve this problem, we follow the arguments in the proof of Theorem 1. First, there exists a nonnegative solution to problem (1.20), since if $u_{\epsilon} \in W^{1,1}(\Omega)$, so is $\left|u_{\epsilon}\right|$, and

$$
|\nabla| u_{\epsilon}||=\left|\nabla u_{\epsilon}\right| .
$$

Let then $u_{\epsilon}$ be a solution of (1.20) which is non negative, then $\sigma_{\epsilon}=\left|\nabla u_{\epsilon}\right|^{\epsilon-1} \nabla u_{\epsilon}$ verifies

$$
\left\{\begin{array}{c}
-\operatorname{div} \sigma_{\epsilon}+a u_{\epsilon}^{\epsilon}=\lambda_{\epsilon}(G) f u_{\epsilon}^{1^{\star}-1} \\
\sigma_{\epsilon} \cdot \nabla u_{\epsilon}=\left|\nabla u_{\epsilon}\right| \\
u_{\epsilon} \geq 0, u_{\epsilon}=0 \text { on } \partial \Omega .
\end{array}\right.
$$


Defining $\tilde{u}_{\epsilon}$ as in the proof of Theorem 1, one sees that $\tilde{u}_{\epsilon}$ is bounded in $W^{1,1+\epsilon}\left(\mathbf{R}^{n}\right)$ and $\sigma_{\epsilon}$ is bounded in $L^{\frac{1+\epsilon}{\epsilon}}(\Omega)$, and then, by extracting subsequences, one obtains

$$
\tilde{u}_{\epsilon} \rightarrow v
$$

in $B V\left(\mathbf{R}^{n}\right), v=0$ outside of $\Omega$, and $\sigma_{\epsilon} \rightarrow \sigma$ in every $L^{q}(\Omega)$, for all $q<\infty$, with $|\sigma| \leq 1$. By passing to the limit, one gets

$$
\left\{\begin{aligned}
-\operatorname{div} \sigma+\operatorname{asign}^{+} u & =\lambda(G) f u^{1^{\star}-1} \\
u & \geq 0 \\
\sigma \cdot \nabla u & =|\nabla u| \text { in } \Omega \\
\tau \cdot \vec{n}(-u) & =u \text { on } \partial \Omega .
\end{aligned}\right.
$$

As in the proof of Theorem 1, one obtains in the same time that

$$
\int_{\Omega} f u^{1^{\star}}=1
$$

and arguing as in the previous section, one can show that $u$ is a nonnegative solution to the relaxed problem (1.11).

\subsection{Existence of nodal solutions}

We are now under the assumptions of Theorem 2:

Theorem 2. Let $\Omega$ be a bounded open set of $\mathbf{R}^{n}$ whose boundary is piecewise $\mathcal{C}^{1}$. Let $s$ be an involution of $\mathbf{R}^{n}$, and $G$ a subgroup of $O\left(\mathbf{R}^{n}\right)$, such that $G$ and $s$ commute weakly, i.e. $O_{G}(s(x))=s\left(O_{G}(x)\right)$. Let $H=[G, s]$ be the subgroup generated by $G$ and $s$. We assume that $\Omega$ is invariant under $H$ as well as $f$ and a, and that $a$ verifies the assumption of coercivity (1.1), that $f$ is positive somewhere. Define

$$
\lambda_{G}^{s}=\inf _{\left\{u \in W^{1,1}(\Omega), u \circ \tau=u, \text { for all } \tau \in G, u \circ s=-u, \int_{\Omega} f|u|^{1^{\star}}=1\right\}}\left\{\int_{\Omega}|\nabla u|+\int_{\Omega} a|u|\right\} .
$$

Assume that for all $x \in \bar{\Omega}$,

$$
\left(\operatorname{Card}_{H}(x)\right)^{\frac{-1}{n}} f(x)^{\frac{1}{1^{\star}}} \lambda_{G}^{s} K(n, 1)<1 .
$$

Then there exists some function $u \in B V(\Omega)$ which is $G$-invariant and s-antisymmetrical such that

$$
\left\{\begin{array}{r}
-\operatorname{div} \sigma+a \operatorname{sign} u=\lambda_{G}^{s} f|u|^{1^{\star}-2} u \\
\left.\left.\sigma \in L^{\infty}\left(\Omega, \mathbf{R}^{n}\right)\right)\right), \sigma \cdot \nabla u=|\nabla u| \\
\sigma \cdot \vec{n} u=-|u| \\
u \text { is } G-\text { invariant and } u \circ s=-u .
\end{array}\right.
$$

Proof. The proof of Theorem 2 follows both [6] and the proof of Theorem 1. We consider the following partial differential equation:

$$
\left\{\begin{array}{c}
-\operatorname{div} \sigma_{\epsilon}+a(x)|u|^{\epsilon-1} u_{\epsilon}=\lambda_{\epsilon}^{s}(G) f(x)|u|^{1^{\star}-2} u_{\epsilon} \text { in } \Omega \\
u \text { is } G-\text { invariant, } u \circ s=-u . \\
\sigma_{\epsilon}=\left|\nabla u_{\epsilon}\right|^{\epsilon-1} \nabla u_{\epsilon} \\
u_{\epsilon} \in W_{0}^{1,1+\epsilon}(\Omega),
\end{array}\right.
$$

where

$$
\lambda_{\epsilon}^{s}(G)=\inf _{\left\{u_{\epsilon} \in W_{0}^{1,1+\epsilon}(\Omega), u \circ \tau=u, \forall \tau \in G, u \circ s=-u \int_{\Omega}, f|u|^{1^{\star}}=1\right\}}\left\{\int_{\Omega}|\nabla u|^{1+\epsilon}+\int_{\Omega} a(x)|u|^{1+\epsilon}\right\} .
$$


This problem possesses a solution that we denote by $u_{\epsilon}$, which satisfies the following equation: $\forall \varphi \in \mathcal{D}(\Omega, \mathbf{R})$ such that $\varphi \circ \tau=\varphi, \forall \tau \in G$, and $\varphi \circ s=-\varphi$

$$
-\int_{\Omega} \operatorname{div} \sigma_{\epsilon} \varphi+\int_{\Omega} a\left|u_{\epsilon}\right|^{\epsilon-1} u_{\epsilon} \varphi=\lambda_{\epsilon}(G) \int_{\Omega} f\left|u_{\epsilon}\right|^{1^{\star}-2} u_{\epsilon} \varphi .
$$

In order to prove that equation (1.42) may be extended to every $\varphi \in \mathcal{D}(\Omega)$, suppose by contradiction that there exists $\varphi \in \mathcal{D}(\Omega)$ such that

$$
-\int_{\Omega} \operatorname{div} \sigma_{\epsilon} \varphi+\int_{\Omega} a\left|u_{\epsilon}\right|^{\epsilon-1} u_{\epsilon} \varphi-\lambda_{\epsilon}^{s}(G) \int_{\Omega} f\left|u_{\epsilon}\right|^{1^{\star}-2} u_{\epsilon} \varphi=\alpha>0
$$

and define

$$
\varphi_{G}^{s}=\varphi_{G}-\varphi_{G} \circ s
$$

where $\varphi_{G}$ has been defined in the proof of Theorem 1. Then $\varphi_{G}^{s}$ is $G$-invariant and $s$-antisymmetrical. By using the $H$-invariance of $\Omega, a$ and $f$, and integrating over $\Omega$ with respect to the Haar measure $d \mu$ on $\Omega$ one obtains

$$
-\int_{\Omega} \operatorname{div} \sigma_{\epsilon} \varphi_{G}^{s}+\int_{\Omega} a\left|u_{\epsilon}\right|^{\epsilon-1} u_{\epsilon} \varphi_{G}^{s}-\lambda_{\epsilon}(G) \int_{\Omega} f\left|u_{\epsilon}\right|^{1^{\star}-2} u_{\epsilon} \varphi_{G}^{s}=2 \alpha
$$

from which we get a contradiction.

Finally, one obtains that $u_{\epsilon}$ is a solution of (1.40). Now, it is not difficult to see that $\overline{\lim }_{\epsilon \rightarrow 0} \lambda_{\epsilon}^{s}(G)=\bar{\lambda}^{s} \leq$ $\lambda_{s}(G)$, as we did in Section 1.2.

Let $\tilde{u}_{\epsilon}$ be the extension of $u_{\epsilon}$ by 0 outside of $\Omega$. Then $\tilde{u}_{\epsilon} \in W^{1,1+\epsilon}\left(\mathbf{R}^{n}\right)$ and is bounded in $W^{1,1}\left(\mathbf{R}^{n}\right)$. Then, up to a subsequence, it converges weakly to some $v \in B V\left(\mathbf{R}^{n}\right)$. By the compactness of the embedding from $W^{1,1}\left(\mathbf{R}^{n}\right)$ into $L^{k}\left(\mathbf{R}^{n}\right)$ for all $k<1^{\star}$, one may assume that $\tilde{u}_{\epsilon}$ tends to $v$ almost everywhere. As a consequence, $v \circ \tau=u, \forall \tau \in G$, and $v \circ s=-v$, i.e. $v$ is $G$-invariant and $s$-antisymmetrical. One can prove as we did for Theorem 1 that $\sigma_{\epsilon}$ tends to some function $\sigma$ in every $L^{q}(\Omega)$ weakly, for every $q<\infty$, such that $\sigma \in L^{\infty}(\Omega)$. Moreover $|\sigma| \leq 1$, and $(v, \sigma)$ verifies the P.D.E

$$
-\operatorname{div} \sigma+a \operatorname{sign} v=\bar{\lambda}^{s}|v|^{1^{\star}-2} v .
$$

Multiplying this equation by $v \phi$ where $\phi \in \mathcal{D}(\bar{\Omega})$, one has:

$$
\int_{\bar{\Omega}} \sigma \cdot \nabla v \phi+\int_{\Omega} \sigma v \cdot \nabla \phi+\int_{\Omega} a|v| \phi=\bar{\lambda}^{s} \int_{\Omega} f|v|^{1^{\star}}
$$

and multiplying (1.40) by $\tilde{u}_{\epsilon} \phi$ one has

$$
\int_{\bar{\Omega}}\left|\nabla \tilde{u}_{\epsilon}\right|^{1+\epsilon} \phi+\int_{\Omega} \sigma \tilde{u}_{\epsilon} \cdot \nabla \phi+\int_{\Omega} a\left|\tilde{u}_{\epsilon}\right|^{1+\epsilon} \phi=\bar{\lambda}^{s} \int_{\Omega} f\left|\tilde{u}_{\epsilon}\right|^{1^{\star}} .
$$

Using Proposition 3, one can assume that there exist two nonnegative and $H$-invariant measures $\mu$ and $\nu$ on $\bar{\Omega}$, a numerable set $\left(x_{i}\right) \subset \bar{\Omega}$ also $H$-invariant, and some non-negative reals $\mu_{i}$ and $\nu_{i}$, such that for all $\varphi \in \mathcal{D}\left(\mathbf{R}^{n}\right)$, one has (by passing to the limit in (1.44))

$$
\langle\mu, \varphi\rangle-\int_{\mathbf{R}^{n}} \sigma . \nabla v \varphi=\bar{\lambda}^{s}\langle\nu, f \varphi\rangle .
$$

Substracting (1.43) from (1.45), one obtains

$$
\int_{\mathbf{R}^{n}}|\nabla v| \varphi-\int_{\mathbf{R}^{n}} \sigma . \nabla v \varphi+\sum_{i} \mu_{i} \varphi\left(x_{i}\right)=\bar{\lambda}^{s} \sum_{i} \nu_{i} \varphi\left(x_{i}\right) f\left(x_{i}\right) .
$$


In addition $\sigma$ verifies:

$$
-\operatorname{div} \sigma+a \operatorname{sign} v=\bar{\lambda}^{s} f|v|^{1^{\star}-2} v
$$

in $\Omega$. We must prove that under the assumption (1.38) in Theorem $2, \mu_{i}=\nu_{i}=0$. For that aim, let $h$ be a $|\nabla v|$-measurable function and $\mu^{\perp}$ be a measure orthogonal to $|\nabla u|$, such that, according to the Radon-Nikodym decomposition, one has

$$
\mu=h|\nabla v|+\mu^{\perp} .
$$

By Lemma 1 and the analogous of (1.34) in 2) of Proposition 3, one has

$$
h|\nabla v| \geq|\nabla v|
$$

and

$$
\mu^{\perp} \geq \sum_{i} \mu_{i} \delta_{x_{i}} .
$$

Using (1.46) and (1.47) in equation (1.45) one gets that

$$
h|\nabla v|=\sigma . \nabla v \text { on } \bar{\Omega}
$$

and

$$
\mu^{\perp}=\sum_{i} \mu_{i} \delta_{x_{i}}=\bar{\lambda} \sum_{i} \nu_{i} f\left(x_{i}\right) \delta_{x_{i}} .
$$

From (1.49), one gets that as soon as $f\left(x_{i}\right)<0$, then $\mu_{i}=\nu_{i}=0$. From (1.47), using $|\sigma| \leq 1$ and Proposition 1, one obtains that in the sense of measures

$$
|\sigma . \nabla v| \leq|\nabla v| \text { on } \bar{\Omega}
$$

and then

$$
\sigma . \nabla v=|\nabla v|
$$

on $\bar{\Omega}$, and $h=1,|\nabla v|$-almost everywhere. Using this in equation (1.45) with $\varphi=1$ one gets that

$$
\int_{\Omega}|\nabla v|+\int_{\partial \Omega}|v|+\int_{\Omega} a|v|=\bar{\lambda} \int_{\Omega} f|v|^{1^{\star}}
$$

By the 1-coercivity of $a$, one obtains that $\int_{\Omega} f|v|^{1^{\star}} \geq 0$, and replacing this in the equation

$$
1=\int_{\Omega} f|v|^{1^{\star}}+\sum_{i} \nu_{i} f\left(x_{i}\right)
$$

we derive that $\sum_{i} \nu_{i} f\left(x_{i}\right) \in[0,1]$ for all $i$. This implies that for all $i$,

$$
\left(f\left(x_{i}\right) \nu_{i}\right) \leq \frac{1}{\operatorname{Card} O_{H}\left(x_{i}\right)}
$$

Indeed, if $\operatorname{Card}_{H}\left(x_{i}\right)<\infty$, suppose by contradiction that there exists $i \in \mathbf{N}$ such that $\left(f\left(x_{i}\right) \nu_{i}\right)>\frac{1}{\operatorname{CardO} O_{H}\left(x_{i}\right)}$. Then, summing over the orbit of $x_{i}$ one obtains a contradiction with

$$
1<\left(\sum_{x_{j} \notin O_{H}\left(x_{i}\right)} f\left(x_{j}\right) \nu_{j}+\sum_{x_{k} \in O_{H}\left(x_{i}\right)} f\left(x_{k}\right) \nu_{k}+\int_{\Omega} f|v|^{1^{\star}}\right) \leq \lim _{\epsilon \rightarrow 0} \int_{\Omega} f\left|u_{\epsilon}\right|^{1 \star}=1 .
$$


Using this inequality one obtains also that as soon as $\operatorname{Card}_{H}\left(x_{i}\right)=\infty, \nu_{i}=0$. We now write, using Proposition 1 and the assumption $\lambda_{G}^{s} f(x)^{\frac{n-1}{n}} K(n, 1) \operatorname{Card}_{H}(x)^{\frac{-1}{n}}<1$ for all $x \in \bar{\Omega}$ :

$$
\begin{aligned}
\mu_{i} & \leq \bar{\lambda}^{s} f\left(x_{i}\right) \nu_{i}=\bar{\lambda}^{s} f\left(x_{i}\right)^{\frac{1}{1^{\star}}} f\left(x_{i}\right)^{1-\frac{1}{1^{\star}}} \nu_{i}^{1-\frac{1}{1^{\star} \star}} \nu_{i}^{\frac{1}{1 \star}} \leq \lambda_{G}^{s}\left(\operatorname{card} O_{H}\left(x_{i}\right)^{-1+\frac{1}{1^{\star}}} f\left(x_{i}\right)^{\frac{1}{1^{\star}}} \nu_{i}^{\frac{1}{1 \star}}\right. \\
& \leq \lambda_{G}^{s}\left(\operatorname{card} O_{H}\left(x_{i}\right)^{-1+\frac{1}{1^{\star}}} f\left(x_{i}\right)^{\frac{1}{1 \star}} K(n, 1) \mu_{i} \leq c \mu_{i}\right.
\end{aligned}
$$

where $c<1$.

This implies that for all $i, \mu_{i}=\nu_{i}=0$, and one concludes as in the previous section.

\section{Estimates AND TEST FUnCTIONS}

Theorem 4. Suppose that $\Omega$ is a bounded open set of $\mathbf{R}^{n}$ whose boundary is piecewise $\mathcal{C}^{1}$, that a and $f$ are two smooth functions on $\bar{\Omega}$, a being 1-coercive and $f$ being positive somewhere, and that $x_{0} \in \Omega$ is such that $f\left(x_{0}\right)>0$. Define

$$
\begin{aligned}
& k_{f}=\inf \left\{k \geq 1, \Delta^{k} f(x) \neq 0\right\}^{1} \\
& k_{a}=\inf \left\{k \geq 0, \Delta^{k} a\left(x_{0}\right) \neq 0\right\} .
\end{aligned}
$$

Then

i) if $k_{a}<k_{f}$, and $\Delta^{k_{a}} a\left(x_{0}\right)<0$,

$$
\lambda(\Omega, a, f)) f\left(x_{0}\right)^{\frac{1}{1^{\star}}} K(n, 1)^{-1+\frac{1}{1^{\star}}}<1 .
$$

ii) If $k_{a} \geq k_{f} \geq 2$ and $\Delta^{k_{f}} f\left(x_{0}\right)>0$ then

$$
\lambda(\Omega, a, f)) f\left(x_{0}\right)^{\frac{1}{1^{\star}}} K(n, 1)^{-1+\frac{1}{1^{\star}}}<1 .
$$

iii) If $k_{f}=1$ and $a\left(x_{0}\right)<0$,

$$
\lambda(\Omega, a, f)) f\left(x_{0}\right)^{\frac{1}{1^{\star}}} K(n, 1)^{-1+\frac{1}{1^{\star}}}<1 .
$$

Proof. Let $u_{\epsilon}$ be the characteristic function of the ball of center $x_{0}$ and radius $\epsilon$, where $\epsilon$ is choosen small enough such that $d\left(x_{0}, \partial \Omega\right)<\epsilon$. The ratio

$$
\frac{\int\left|\nabla u_{\epsilon}\right|}{\left(\left|B\left(x_{0}, \epsilon\right)\right|^{\frac{n-1}{n}}\right)}
$$

has value $K(n, 1)$. Let us define $J\left(u_{\epsilon}\right)=\frac{\int_{\Omega}\left|\nabla u_{\epsilon}\right|+\int_{B\left(x_{0}, \epsilon\right)} a u_{\epsilon}}{\left(\int_{B\left(x_{0}, \epsilon\right)} f u_{\epsilon}^{1 \star}\right)^{\frac{n-1}{n}}}$. We shall prove, developing $a$ and $f$ around $x_{0}$, that for $\epsilon$ small, $J\left(v_{\epsilon}\right)<\frac{1}{K(n, 1) f\left(x_{0}\right)^{1-\frac{1}{n}}}$. We write for every $J \in \mathbf{N}$

$$
\int_{B\left(x_{0}, \epsilon\right)} a(x) d x=\sum_{|j| \leq J} \frac{D^{j} a\left(x_{0}\right)}{j !} \int_{B\left(x_{0}, \epsilon\right)} x^{j} d x+o\left(\epsilon^{n+J}\right)
$$

where $j=\left(j_{1}, . . j_{n}\right),|j|=j_{1}+. . j_{n}, D^{j}=\frac{\partial^{|j|}}{\partial_{1}^{j_{1}} \partial_{n}^{j_{n}}}$ and $x^{j}=x_{1}^{j_{1}} x_{2}^{j_{2}} . . x_{n}^{j_{n}}$ Arguing as in [6], Lemma 6, one has for all $J$ and $K$

$$
\begin{aligned}
& \int_{B\left(x_{0}, \epsilon\right)} a(x) d x=\left|S_{n-1}\right| \sum_{j \leq J} \epsilon^{n+2 j} \frac{\Delta^{j} a\left(x_{0}\right)}{j !} \alpha_{n, j}+O\left(\epsilon^{n+2 J}\right), \\
& \int_{B\left(x_{0}, \epsilon\right)} f(x) d x=\left|S_{n-1}\right| \sum_{k \leq K} \epsilon^{n+2 k} \alpha_{n, k} \frac{\Delta^{k} f\left(x_{0}\right)}{k !}+O\left(\epsilon^{n+2 K}\right),
\end{aligned}
$$

\footnotetext{
${ }^{1} \Delta^{k}$ denotes the $k$-iterate of the Laplacian.
} 
where

$$
\alpha_{n, j}=\frac{\Gamma\left(j+\frac{1}{2}\right) \Gamma\left(\frac{1}{2}\right)^{n-1}}{\Gamma\left(j+\frac{n}{2}+1\right)}
$$

One obtains

$$
\begin{aligned}
& \frac{\int\left|\nabla u_{\epsilon}\right|+\int_{B\left(x_{0}, \epsilon\right)} a u_{\epsilon}}{\left(\int_{B\left(x_{0}, \epsilon\right)} f\right)^{\frac{n-1}{n}}}=\frac{n^{1-\frac{1}{n}}\left|S_{n-1}\right|^{\frac{1}{n}}}{f\left(x_{0}\right)^{1-\frac{1}{n}}}\left(1+\sum_{j \leq J} \epsilon^{1+2 j} \frac{\alpha_{n, j}}{j !} \frac{\Delta^{j} a\left(x_{0}\right)}{\left|S_{n-1}\right|}+O\left(\epsilon^{2+2 J}\right)\right) \\
& \left(1+\sum_{k \leq K} \frac{n \epsilon^{2 k} \alpha_{n, k} \Delta^{k} f\left(x_{0}\right)}{\left.\left|S_{n-1}\right| k ! f\left(x_{0}\right)\right)^{1 / n-1}}+O\left(\epsilon^{2 K+1}\right)\right)^{-1+\frac{1}{n}} .
\end{aligned}
$$

Suppose now that $k_{a}<k_{f}$, then $2 k_{a}+1<2 k_{f}$, and the first non zero term to consider in the expansion of powers of $\epsilon$ above is $\epsilon^{2 k_{a}+1} \frac{\alpha_{n, k_{a}} \Delta^{k_{a}} a\left(x_{0}\right)}{k !}$. Hence the result follows as soon as $\Delta^{k_{a}} a\left(x_{0}\right)<0$.

Suppose that $k_{a} \geq k_{f} \geq 2$ and $\Delta^{k_{f}}\left(x_{0}\right)>0$. Then the first non zero term in the expansion of powers of $\epsilon$ above is $\left(-1+\frac{1}{n}\right) \frac{n \epsilon^{2 k} \alpha_{n, k} \Delta^{k} f\left(x_{0}\right)}{\left.\left|S_{n-1}\right| k ! f\left(x_{0}\right)\right)^{1 / n-1}}$ which is strictly negative. The result follows.

\section{Some SPECIFIC EXAMPLES}

We present in this section some concrete situations where the results of the preceding section can apply:

Proposition 3.1. Assume that $\Omega$ is a bounded ball of center 0 and radius $r>0$. Suppose that one of of the three following situations occurs:

- $k_{f}(0)=1$ and $a(0)<0$,

- $k_{f}(0) \geq 2, k_{a}(0)<k_{f}(0)$ and $\Delta^{k_{a}} a(0)<0$,

- $k_{f}(0) \geq k_{a}(0)$ and $\Delta^{k_{f}}(0)>0$.

Then there exists a radial solution to the relaxed problem (1.11).

Proposition 3.2. Assume that $a=0$ and $f$ is not constant and positive somewhere. Suppose that there exists $x_{0} \in \Omega$ on which $f$ achieves its maximum, and that $\Delta^{k_{f}} f\left(x_{0}\right)>0$. (necessarily $k_{f}>1$ ). Then, there exists a solution to the problem

$$
\begin{aligned}
-\operatorname{div} \sigma+a(x) \operatorname{sign} u & =f|u|^{1^{\star}-2} u \\
\sigma . \nabla u & =|\nabla u|
\end{aligned}
$$

$u$ is not identically zero, $-\sigma . n u=|u|$ on $\partial \Omega$.

Proposition 3.3. Assume that $\Omega$ is a solid torus of $\mathbf{R}^{3}$, obtained by rotation around the $z$-axis, let $s$ be the orthogonal symmetry with respect to the $(x, y)$ plane, and let $H=[G, s] ;$ Let also a and $f$ be smooth on $\bar{\Omega}, f$ being positive somewhere, a being such that (1.1) is satisfied. Then the problem

$$
\left\{\begin{aligned}
-\operatorname{div} \sigma+a(x) \operatorname{sign} u & =f|u|^{1^{\star}-2} u \\
\sigma . \nabla u & =|\nabla u| \\
u \text { is not identically zero, }, & -\sigma . n u=|u| \text { on } \partial \Omega
\end{aligned}\right.
$$

possesses a nonnegative $G$-invariant solution $u \in B V(\Omega)$, and a nodal solution $G$-invariant and s-antisymmetrical solution $u \in B V(\Omega)$, whose zero set is exactly the intersection of $\Omega$ with the $(x, y)$ plane. 
Proposition 3.4. Let $\Omega$ be an annulus of $\mathbf{R}^{n}$ centered at 0 , and $a<0$ a real number such that (1.1) is satisfied. Then problem (1.11) has a solution which satisfies:

$$
\left\{\begin{aligned}
-\operatorname{div} \sigma+a(x) \operatorname{sign} u & =\lambda_{s}^{G} f|u|^{1^{\star}-2} u \\
\sigma . \nabla u & =|\nabla u| \\
u \text { is not identically zero, } & -\sigma \cdot n u=|u| \text { on } \partial \Omega
\end{aligned}\right.
$$

which is radial, and nonnegative. Moreover, this problem possesses infinitely many nodal solutions.

Proof of Proposition 3.4. Let $G=O\left(\mathbf{R}^{n}\right)$. Since for every $x \neq 0, \operatorname{card} O_{G}(x)=\infty$, one gets by Theorem 1 that (1.11) possesses a nonnegative radial solution. For the second assertion of the Theorem, let $P$ be some hyperplan of $\mathbf{R}^{n}$ such that $0 \in P$, and let $D_{P}$ be the orthogonal complement of $P, 0 \in D_{P}$. We denote by $G_{P}$ the group of rotations around $D_{P}$ and by $s_{P}$ the orthogonal symmetry with respect to $P$. In order to verify the sufficient condition (1.37) in Theorem 2, we distinguish two cases: either $x_{0} \in D_{P} \cap \Omega$ and then $\operatorname{card}_{H}\left(x_{0}\right)=\operatorname{card}_{G}\left(x_{0}\right)=2$. Then the assumptions on $a$ and $f$ and Proposition 3.1 imply that $\lambda_{G}^{s}<\frac{2^{\frac{1}{n}}}{K(n, 1)}$. If $x_{0} \in D_{P} \cap \Omega, \operatorname{card} O_{G}\left(x_{0}\right)=\infty$ and condition (1.37) is once more verified. By Theorem 2, one gets the existence of a nodal solution $u_{P} \in B V(\Omega)$. In particular, the properties of symmetry of $u_{P}$ imply that $u_{P_{1}} \neq u_{P_{2}}$ (in the sense of distributions for example). Since $P$ is arbitrary, this proves the second part of the proposition.

\section{The analogous of Pohö̈aev identity}

Theorem. Let $\Omega$ be a bounded open set of $\mathbf{R}^{n}$ which is piecewise $\mathcal{C}^{1}$. Let $g$ be a continuous function from $\mathbf{R}$ into $\mathbf{R}$, and let $G$ be its antiderivative which satisfies $G(0)=0$. Suppose that $u \in W_{\text {loc }}^{2,1}(\Omega) \cap W_{0}^{1,1}(\Omega)$ is a solution to the problem:

$$
\left\{\begin{aligned}
-\operatorname{div} \sigma & =g(u) \\
u & =0 \text { sur } \partial \Omega \\
\sigma . \nabla u & =|\nabla u| \\
u & \geq 0 \text { on } \Omega .
\end{aligned}\right.
$$

Then

$$
(n-1) \int_{\Omega}|\nabla u|=n \int_{\Omega} G(u) .
$$

In particular, one cannot conclude that $u=0$ in the case where $g(u)=u^{1^{\star}-1}$.

Proof. Let $\varphi_{0}$ be in $\mathcal{C}^{\infty}\left(\left[0, \infty[)\right.\right.$ such that $\varphi_{0}=1$ on $] 0,1\left[\right.$, and $\varphi=0$ for $|t| \geq 2$. Let $j \in \mathbf{N}$, and $\varphi_{j}$ is defined on $\mathbf{R}^{N}$ by $\varphi_{j}(x)=\varphi_{0}\left(\frac{|x|}{j}\right)$. Then

$$
|x|\left|\nabla \varphi_{j}(x)\right| \leq \frac{|x|}{j}\left|\varphi_{0}^{\prime}\left(\frac{|x|}{j}\right)\right| \leq \frac{C}{j}\left\|\varphi_{0}^{\prime}\right\|_{\infty}
$$

with $C$ some constant independent of $j$.

Let us multiply the equation by $\left(x_{i}-x_{i}^{\star}\right) \varphi_{j} u_{, i}$. One obtains after integrating by parts on the right hand side:

$$
\int_{\Omega} g(u)\left(x_{i}-x_{i}^{\star}\right) \varphi_{j} u_{, i}=-\int_{\Omega} G(u) \partial_{i}\left(\left(x-x_{i}^{\star}\right) \varphi_{j}\right)=-\int_{\Omega} G(u) \varphi_{j}-\int_{\Omega} G(u)\left(x_{i}-x_{i}^{\star}\right) \varphi_{j, i}
$$

since $G(0)=0$ and $u=0$ on $\partial \Omega$. The last integral tends to zero when $j$ goes to infinity. We now treat the integral on the left hand side:

$$
\int_{\Omega}-\operatorname{div} \sigma\left(x_{i}-x_{i}^{\star}\right) \varphi_{j} u_{, i}=\int_{\Omega} \sigma_{i} u_{, i} \varphi_{j, i}+\int_{\Omega} \sigma_{k} u_{, i k}\left(x_{i}-x_{i}^{\star}\right) \varphi_{j}-\int_{\partial \Omega} \sigma \cdot \vec{n}\left(x_{i}-x_{i}^{\star}\right) \varphi_{j} u_{, i} .
$$


By integrating by parts once more, one obtains

$$
\begin{aligned}
\int_{\Omega} \sigma_{k} u, i k\left(x_{i}-x_{i}^{\star}\right) \varphi_{j} & =\int_{\Omega} \partial_{i}(|\nabla u|)\left(x_{i}-x_{i}^{\star}\right) \varphi_{j}+\int_{\partial \Omega}|\nabla u|\left(x_{i}-x_{i}^{\star}\right) \vec{n}_{i} \varphi_{j} \\
& =-\int_{\Omega}|\nabla u| \varphi_{j}-\int_{\Omega}|\nabla u|\left(x_{i}-x_{i}^{\star}\right) \varphi_{j, i}+\int_{\partial \Omega}|\nabla u|\left(x_{i}-x_{i}^{\star}\right) \vec{n}_{i} \varphi_{j} .
\end{aligned}
$$

One can remark that the integrals $\int_{\Omega}|\nabla u|\left(x_{i}-x_{i}^{\star}\right) \varphi_{j, i}$ and $\int_{\Omega} \sigma_{i} u_{, i} \varphi_{j, i}$ tend to zero when $j$ goes to infinity. By passing to the limit when $j$ goes to infinity, summing over $i$, using $\int_{\Omega}|\nabla u|=\int_{\Omega} u^{1^{\star}}$ and the fact that since $u=0$ on $\partial \Omega, \nabla u$ is parallel to $\vec{n}$ on $\partial \Omega$, one finally gets the identity:

$$
(n-1) \int_{\Omega}|\nabla u|=-n \int_{\Omega} G(u) .
$$

When $g(u)=u^{1^{\star}-1}$, one obtains:

$$
(n-1) \int_{\Omega}|\nabla u|=\frac{n}{1^{\star}} \int_{\Omega} u^{1^{\star}}=(n-1) \int_{\Omega}|\nabla u| .
$$

We have no contradiction as it was the case for the equations

$$
\begin{gathered}
-\operatorname{div}\left(|\nabla u|^{p-2} \nabla u\right)=u^{p^{\star}-1} \\
u \in W_{0}^{1, p} \cap W_{l o c}^{2, p}(\Omega)
\end{gathered}
$$

with $p>1$.

\section{REFERENCES}

[1] T. Aubin, Problèmes isopérimétriques et espaces de Sobolev. J. Differential Geom. 11 (1976) 573-598.

[2] T. Aubin, Nonlinear Analysis on manifolds-Monge-Ampère Equations. Grundlehern der Mathematischen Wissenschaften (1982) 252.

[3] A. Bahri and J.M. Coron, On a non linear elliptic equation involving the critical Sobolev exponent: The effet of the topology of the domain. Comm. Pure Appl. Math. 41 (1988) 253-294.

[4] Bobkov and Ch. Houdré, Some connections between isoperimetric and Sobolev type Inequalities. Mem. Amer. Math. Soc. 616 (1997).

[5] F. Demengel, Some compactness result for some spaces of functions with bounded derivatives. Arch. Rational Mech. Anal. 105 (1989) 123-161.

[6] F. Demengel and E. Hebey, On some nonlinear equations involving the $p$-Laplacian with critical Sobolev growth. I. Adv. Partial Differential Equations 3 (1998) 533-574.

[7] I. Ekeland and R. Temam, Convex Analysis and variational problems. North-Holland (1976).

[8] E. Giusti, Minimal surfaces and functions of bounded variation, notes de cours rédigés par G.H. Williams. Department of Mathematics Australian National University, Canberra (1977), et Birkhaüser (1984).

[9] E. Hebey, La méthode d'isométrie concentration dans le cas d'un problème non linéaire sur les variétés compactes à bord avec exposant critique de Sobolev. Bull. Sci. Math. 116 (1992) 35-51.

[10] E. Hebey and M. Vaugon, Existence and multiplicity of nodal solutions for nonlinear elliptic equations with critical Sobolev Growth. J. Funct. Anal. 119 (1994) 298-318.

[11] P.L. Lions, La méthode de compacité concentration, I et II. Revista Ibero Americana 1 (1985) 145.

[12] R.V. Kohn and R. Temam, Dual spaces of stress and strains with applications to Hencky plasticity. Appl. Math. Optim. 10 (1983) 1-35.

[13] B. Nazaret, Stability results for some nonlinear elliptic equations involving the $p$-Laplacian with critical Sobolev growth, COCV, accepted Version française : Prepublication de l'Université de Cergy-Pontoise N 5/98, Avril 1998.

[14] Talenti, Best constants in Sobolev inequality. Ann. Mat. Pura Appl. (4) 110 (1976) 353-372.

[15] G. Strang and R. Temam, Functions with bounded variations. Arch. Rational Mech. Anal. (1980) 493-527.

[16] P. Suquet, Sur les équations de la plasticité. Ann. Fac. Sci. Toulouse Math. (6) 1 (1979) 77-87.

[17] Ziemmer, Weakly Differentiable functions. Springer Verlag, Lectures Notes in Math. 120 (1989). 